\title{
A CLOSED-FORM APPROACH TO PREDICT GUST INDUCED FATIGUE DAMAGES
}

\author{
Frank H. Kemper ${ }^{2}$ \\ ${ }^{1}$ Institute for Steel Structures \\ RWTH Aachen University \\ 52070 Aachen \\ e-mail: frank.kemper@rwth-live.de
}

Keywords: Structural Dynamics, Wind Engineering, Slender Structures, Stochastic Methods, Fatigue

\begin{abstract}
The investigation of gust induced dynamic responses of structures is still subject of current research in wind engineering. Especially with regard to the fatigue life prognosis, different methods have been discussed in recent years - but all current design codes still have not implemented a general reliable guidance for the determination of stress-range spectra and the application of cumulative damage calculations. The present paper makes use of spectral methods for the determination of responses to stochastic loading and shows, that based on power spectral densities of the structural responses, reliable approximations of the Rainflow stressrange spectra are possible. Consequently, fatigue verifications are possible by means of closed form spectral methods. The reliability has been proven, compared to time-domain solutions. Thereby the bandwidth of the structural response does not affect the prediction quality. Due to the tight manageability of the presented formulas, the application in all cases, where gust excited structural responses may be significant, is advised. The second part of this paper additionally implements an approximative consideration of nonlinear structural behaviour in the closed form solution methodology.

Convolving with site related wind statistics, realistic predictions of the lifetime related distribution of stress cycles are enabled. Based on the presented methodology, an extensive parameter study has been perfomed in order to discover the dependencies between structural and aerodynamic properties on the resulting stress range distributions. Finally, a simplified approach is presented, which suggests damage equivalence factors, taking into account an individual and realistic shape of the cycle count distribution.

Based on the presented approach, both exact life time predictions and simplified estimations are enabled for wind excited structures. The presented methodoly is demonastrated using the example of a wind turbine tower. For these structures the cyclic loading induced by the wind gust directly into the tower and by the rotor are decisive for the design of the steel structure.
\end{abstract}




\section{Introduction}

Wind affected structures generally have to withstand fluctuating load cycles. For an appraisement whether the resulting stress range spectra may lead to structural failures during the designed lifetime, standard methods are still rare because the influencing parameters are manifold. A realistic determination of structural stress range spectra provoked by the natural wind, requires profound knowledge about the statistical properties of the wind flow at the considered site, as well as about the dynamic structural behavior. Eventually, the interpretation of the resulting structural responses in terms of stress amplitudes $\Delta \sigma$ and associated frequencies of occurrence $N(\Delta \sigma)_{\text {Life }}$ over the expected lifetime are the main task to allow fatigue verifications.

\section{APPROXIMATIVE CLOSED FORM SOLUTIONS}

The statistical description of the wind flow succeeds in two time categories, to consider both, the short-termed flow fluctuations which may lead to resonant structural responses and the longtermed fluctuations, which mainly influence the load level at which structural vibrations occur over the expected life-time. Accordingly, the fatigue relevant loading over the lifetime can be expressed as a combination of the short-termed dynamic effects at a mean load level, which is influenced by the long-termed average flow conditions. With respect to possible resonant wind effects under extreme wind conditions, site related power spectral densities (PSD) of the wind velocity are needed.

Already 50 years ago, Davenport [2] laid the foundation and introduced the probabilistic theory for a usage in the field of wind engineering. Meanwhile nearly all wind codes in the world adopted procedures which are based on his early work for the rating of extreme (short-termed) gust effects. Concerning the long-termed changes of the wind velocity, the probability density function (PDF), e.g. using the two-parametric Weibull distribution, allows the determination of the wind performance under usual conditions.

Structural system properties are generally described by the mechanical admittance function $H(i \omega)$, whereat in most cases a simplified consideration of single degree of freedom systems deems to be sufficient. Decisive for the susceptibility to wind induced resonant vibrations and the associated bandwidth of the structural response, are the natural frequency and the damping ratio of the system. Namely, with increasing structural frequency, the excited responses decrease as a consequence of the falling spectral density, furthermore the response becomes wider by reason of the quasi-static range in the structural admittance function up to the natural frequency.

For the interpretation of structural responses with respect to fatigue, the rain-flow counting method is admittedly the best instrument [17]. The procedure has been developed by Matsuishi and Endo [12] for the application with time histories of stress and can therefore not be used directly in frequency based analyzes. Hence, during the last decades a lot of research effort has been focussed on the finding of approximative solutions.

\section{PSD BASED CYCLE COUNTING}

Although the numerical rainflow method is an efficient, reliable and widely accepted tool for determining the amplitude distribution of stochastic responses, it requires the existence of plausible stress time series. In case of a non-linear structural behavior a fatigue life prognosis requires a considerable diversity of time series which belong to different wind speeds.

For the above reasons, it is more desirable that the determination of the rainflow classifi- 


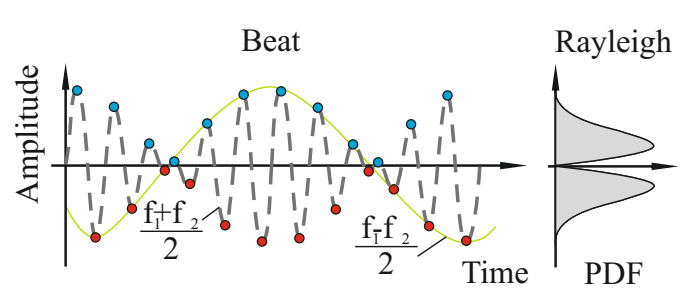

(a) Narrow-band Signal $(\gamma \approx 1)$

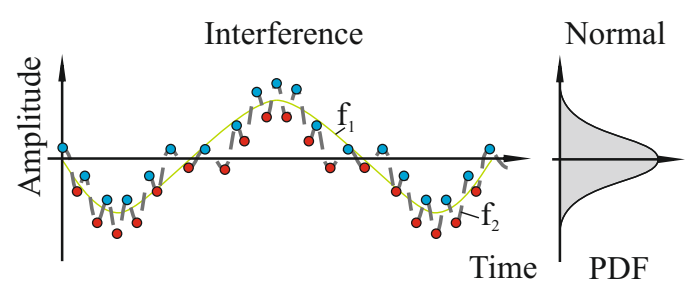

(b) Wide-band Signal $(\gamma \approx 0)$

Figure 1: Visualization of Time Series with different Bandwidths and Effect on the Distribution of Extremes

cations of stochastic processes is enabled in the frequency domain. This task has been extensively treated in recent decades by researchers from different disciplines, especially in aviation and space technology. In the following sections different approaches are presented (which are partially described in [6]) and evaluated in the light of the particular problem of gust excited structural responses. All methods have in common that they do not consider an influence of the mean stress amplitude, which is in accordance e.g. with Eurocode 3-1-9 [4].

The initial point of most stochastic fatigue methods represents the level crossing formula from Rice according to Eq. (1). It describes the density of the positive crossings of a given amplitude level $\sigma$ based on the standard deviation $\sigma_{\sigma}$ of the process to:

$$
f(\sigma)=\frac{\sigma}{\sigma_{\sigma}^{2}} \cdot \exp \left(-\frac{\sigma^{2}}{2 \sigma_{\sigma}^{2}}\right)
$$

For the determination of stress range collectives, an extension has been proposed by Bendat. Using the spectral moments of a response power spectral density $S_{a a}(f)$ :

$$
m_{i}=\int_{0}^{\infty} f_{i} \cdot S_{a a}(f) d f
$$

The cycle rate $E_{P}$ and the rate of the positive level crossings $E_{0}$ (as expected value of the frequency $\nu$ ) is calculated as:

$$
E_{0}=\nu=\sqrt{\frac{m_{2}}{m_{0}}} \quad E_{P}=\sqrt{\frac{m_{4}}{m_{2}}}
$$

Finally an approach for estimating the probability density of the collective stress can be derived [1]. The Bendat solution takes into account the rate of the positive extremes associated with the distribution of these extreme values. It is assumed, that the distribution of the extreme values of a narrowband process can be described by a Rayleigh distribution. Since the number of closed stress cycles are relevant for the fatigue design, the double stress amplitude $\Delta \sigma$ is used for the calculation. Consequently, the estimate of the stress range cycles of a narrow-band process is given by:

$$
N(\Delta \sigma)=\nu \cdot T \cdot f_{r}\left(\Delta \sigma, \sigma_{\Delta \sigma}\right)=\nu \cdot T \cdot \frac{\Delta \sigma}{\sigma_{\Delta \sigma}^{2}} \cdot \exp \left(\frac{-\Delta \sigma^{2}}{2 \cdot \sigma_{\Delta \sigma}^{2}}\right)
$$

Under gusty wind load narrow-band responses can be expected for low frequent structures and weakly damped systems. A distinction between narrow-band and wide-band signals is shown in Fig. 1(a) and Fig. 1(b). With increasing bandwidth of the signal, the presented methodology fails to calculate appropriate level crossings. With respect to structural responses 
of different dynamic systems, alternative methods are needed in order to describe the frequency distribution of the extreme values. The Rice formula overestimates in these cases the actual stress range distributions significantly.

Especially in the field of mechanical engineering an empirical approach for estimating the Rainflow distribution based on the power spectral density is meanwhile well established. Dirlik derived this method based on continuous random processes and subsequent numerical Rainflow counting [3]. He discovered an empirical relationship between the frequency range description of the signal and the Rainflow distribution.

His studies were directed to stationary, ergodic random processes with arbitrary bandwidth. Consistently, the Dirlik probability density function of peak values of a process with any bandwidth is a weighted sum of a normal distribution and a Rayleigh distribution. In previous studies of Wirsching and Shehata [18], a similar approach was chosen, but there an attempt was made to describe the Rainflow distributions by Weibull functions. The simulations of Dirlik, as well as own simulations showed, that the Rainflow distributions of peaks often differs significantly from a Weibull distribution. For this reason, the Wirsching-Factor, which has been used for instance from Holmes [7], is not realistic in case of higher response bandwidth [9].

With Dirlik's formula, the density of the number of cycle counts $N^{\prime}$ of a given stress level $\Delta \sigma$ can be computed to:

$$
\begin{aligned}
& N^{\prime}(\Delta \sigma)=E_{P} \cdot T \cdot f_{D}(\Delta \sigma) \\
& f_{D}(\Delta \sigma)=\frac{\frac{D_{1}}{Q} \cdot e^{\frac{-Z(\Delta \sigma)}{Q}}+\frac{D_{2} \cdot Z(\Delta \sigma)}{R^{2}} \cdot e^{\frac{-Z(\Delta \sigma)^{2}}{2 \cdot R^{2}}}+D_{3} \cdot Z(\Delta \sigma) \cdot e^{\frac{-Z(\Delta \sigma)^{2}}{2}}}{2 \cdot \sqrt{m_{0}}}
\end{aligned}
$$

where:

$$
\begin{array}{rlrl}
x_{m} & =\frac{m_{1}}{m_{0}} \cdot \sqrt{\frac{m_{2}}{m_{4}}} & R & =\frac{\gamma-x_{m}-D_{1}^{2}}{1-\gamma-D_{1}+D_{1}^{2}} \\
D_{1} & =\frac{2 \cdot\left(x_{m}-\gamma^{2}\right)}{1+\gamma^{2}} & D_{2} & =\frac{1-\gamma-D_{1}+D_{1}^{2}}{1-R} \\
D_{3} & =1-D_{1}-D_{2} & Z(\Delta \sigma) & =\frac{\Delta \sigma}{2 \cdot \sqrt{m_{0}}} \\
Q & =\frac{1.25 \cdot\left(\gamma-D_{3}-D_{2} \cdot R\right)}{D_{1}}
\end{array}
$$

are auxillary values.

The accuracy of the method has been verified with respect to gust excited structures by comparing the results to corresponding transient simulations. In all cases, the discrepancies between transient Rainflow count and Dirlik formula where negligible [10]. Even geometrical nonlinear structures can be analyzed based on the presented spectral approach. The necessary stochastic description of the mechanical system has been presented in [11].

\section{FATIGUE BEHAVIOUR AND STRUCTURAL INFLUENCE ON THE LIFETIME}

\subsection{Assessment of Gust Repsonse with the $N_{L i f e}$-Formula}

Making use of the presented PSD based cycle count algorithm, the assessment of stress cycles is enabled. But so far, only the impact of a short-termed extreme wind on the stress 
range distribution has been considered. For the computation of the expected cycles during the designated life-time with respect to fatigue phenomena, the prevalent wind events during this time period have to be taken into account as well.

Hence, the resulting cycle count spectrum during the foreseen lifetime originates from a convolution of short termed stress range spectra and a long-termed wind statistics. It can be computed by the following expression:

$$
N_{\text {Life }}^{\prime}(\Delta \sigma)=\int_{0}^{\bar{u}} \frac{T_{\text {Life }}}{T_{\text {ref }}} \cdot f(u) \cdot N^{\prime}(\Delta \sigma, u) d u
$$

where: $T_{\text {Life }}$ is the designed Lifetime, $T_{r e f}$ is the average time of the mean wind speed, $f(u)$ is the probability density function of the wind velocity and $N(\Delta \sigma, u)$ is the stress range spectra for a given mean wind speed within the average time period. The latter expression is computed based on the Dirlik formula.

The probability density function for the frequency of occurrence of mean wind speeds can be derived based on the Weibull-Distribution:

$$
f(\bar{u})=\frac{k}{A}\left(\frac{\bar{u}}{A}\right)^{k-1} \exp \left[-\left(\frac{\bar{u}}{A}\right)^{k}\right]
$$

where: $A$ is the scale and $k$ is the form parameter of the distribution. Site dependent values for these parameters can be taken from the European Wind Atlas [16].

Based on appropriate assumptions for the site dependent extreme wind impact and the probability of exceedance of the prevalent wind speeds, a realistic consideration of fatigue impact is possible. Using the direction related Weibull Parameter, even a directional design is possible. Finally, the fatigue verification succeeds based on an appropriate damage accumulation approach in conjunction with a suitable s-N-Curve. For example, using a elementary MinerRule:

$$
D=\int_{\Delta \sigma} \frac{N_{L i f e}^{\prime}(\Delta \sigma)}{N_{c} \cdot\left(\frac{\Delta \sigma_{c}}{\Delta \sigma}\right)^{m}} d \Delta \sigma
$$

It is important to consider, that the cycle count density $N_{L i f e}^{\prime}(\Delta \sigma)$ has to be integrated over the range of possible stress amplitudes in order to evaluate the cumulative number of cycles and hence, the total damage.

\subsection{Shape of Cycle Count Distribution and influencing Parameters}

Due to the analytical formulation of Eq. (7), parametric studies of the relation between structural and aerodynamic characteristics on the resulting cycle count distribution can be studied comparatively easy. The described procedure is significantly faster than time-domain methods, which require wind field generations and transient dynamic analyzes. Nevertheless, in case of linear structural systems, the computation succeeds without limitations.

In order to allow a general usage of the cycle count distribution, a normalization of the curve is necessary. It is postulated, that the maximum stress amplitude $\Delta \sigma_{\max }$ occurs exactly once in the designated lifetime. This demand requires the consideration of the cumulative function $N_{\text {Life }}$, as only in this notation a frequency of occurence of $N=1$ can be found. The cumulative 


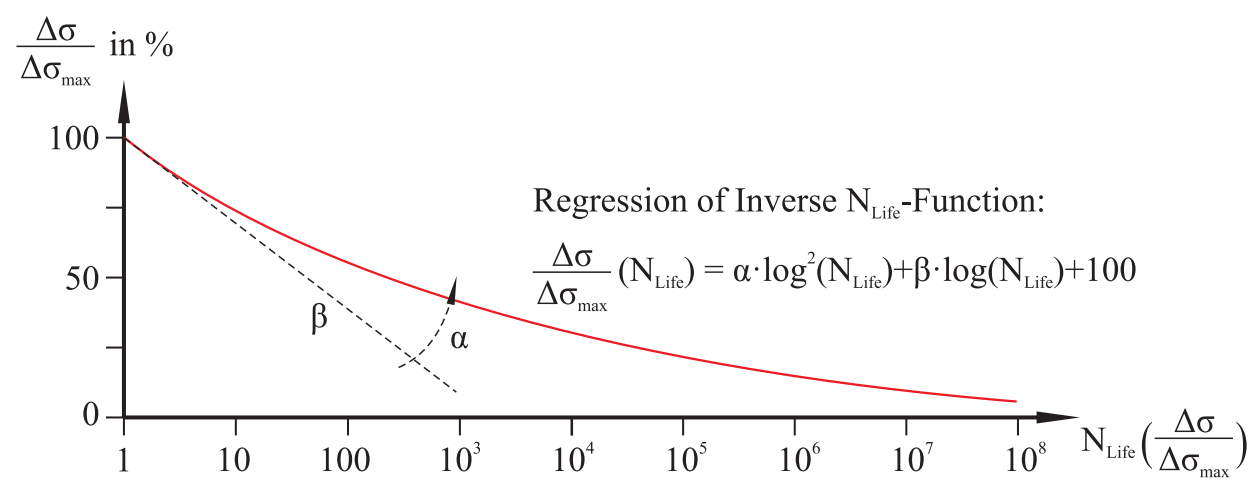

Figure 2: Regression of the Cycle Count Distribution

function is defined as:

$$
N_{\text {Life }}(s)=\int_{0}^{s} N_{\text {Life }}^{\prime}(t) d t
$$

As a result, the $N_{\text {Life }}$-Function allows a quantifiable description of the influences on the damage life. The individual cumulative stress cycle count distributions can now be normalized by the maximum amplitude $\Delta \sigma_{\max }$ at $N=1$ and finally be fitted by a logarithmic polynomial $2^{\text {nd }}$ order:

$$
\frac{\Delta \sigma}{\Delta \sigma_{\max }}\left(N_{\text {Life }}\right)=\alpha \cdot \log ^{2} N_{\text {Life }}+\beta \cdot \log N_{\text {Life }}+100
$$

where the normalized stress $\Delta \sigma / \Delta \sigma_{\max }$ is given in percent. A general distinction has to be made concerning parameters, which affect the amplitude (scaling) of the cycle count distribution and those which affect the distribution (shape). Concerning the first, the normalization of stress amplitude to its maximum separates scaling from shape. Concerning the second, the variation in shape is expressed in the polynomial coefficients $\alpha$ and $\beta$.

\subsubsection{Location}

The of the Weibull parameters $A$ and $k$ on the fatigue loading of structures was desribed by Höffer and Görnandt [15]. Assuming a quasi-static structural behavior, they showed how different Weibull distributions of wind speeds at German sites affect the cumulative number of cycles. They discovered that this influence is significant and a that a simplified, unique assumption for the shape of cumulative cycle counts like in Eurocode 1 is not realistic.

Stochastic calculations have been performed in order to identify the influence of different aerodynamic related parameters on the shape of the cycle count distribution. For these investigations, a designated lifetime of $T_{\text {Life }}=50$ a has been assumed. As structural system a single degree of freedom oscillator with a natural frequency of $f=1.0 \mathrm{~Hz}$ and a logarithmic damping decrement of $\delta=0.01$ has been chosen. The cycle count density $N^{\prime}(\Delta \sigma, u)$ has been determined based on the Dirlik formula according to Eq. (6) at a given level of mean wind speed $u$ and for a reference period of $T_{\text {ref }}=3600 \mathrm{~s}$.

Different aerodynamic values have been modified in order to evaluate the influence on the fatigue life. It has been discovered, that the basic wind speed on site $u_{b}$, the turbulence intensity $I_{u}$, the integral length scale $L_{u x}$ and the quasi-static force coefficient affect mainly the scaling 


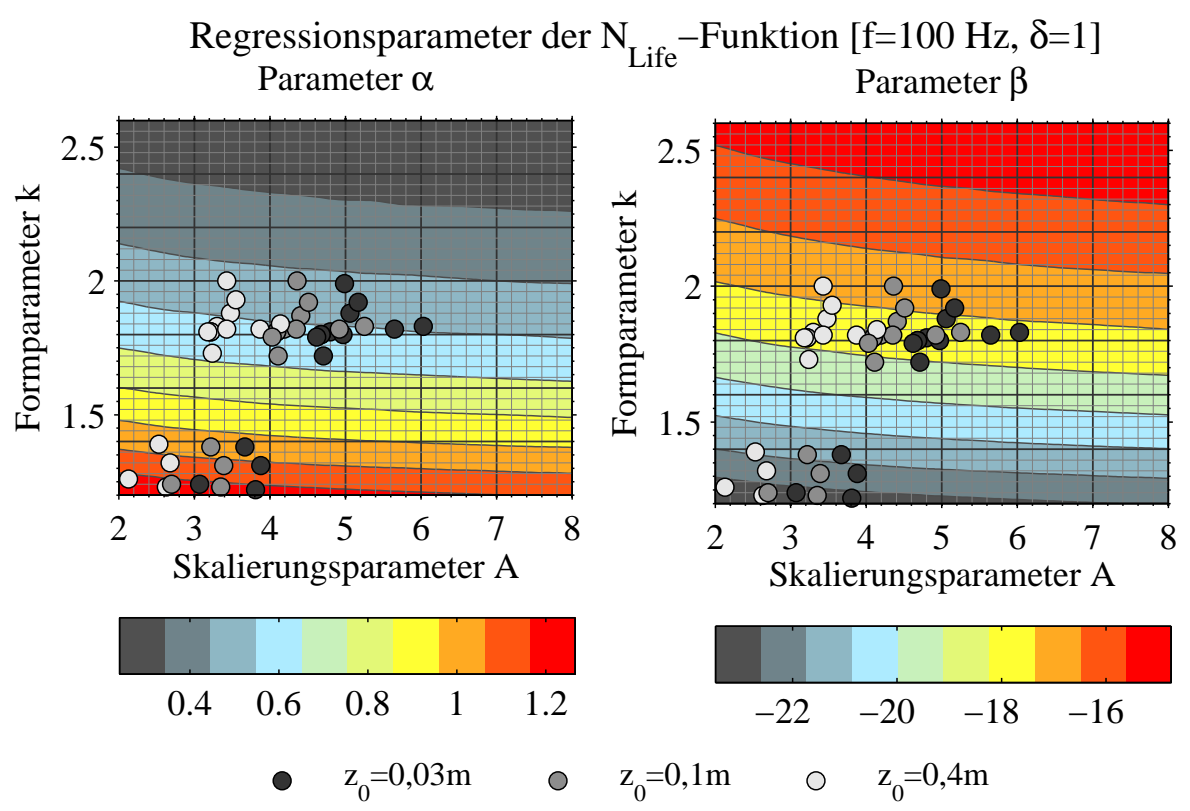

Figure 3: Regression of the $N_{L i f e}$-Curve dependent on the Shape and Scaling Parameters of the Weibull Distribution and Visualization of 15 different Locations in Germany with varying Roughness Length $z_{0}$

of the cycle count distribution [8]. Hence, all mentioned parameters primarily affect the maximum stress amplitude $\Delta \sigma_{\max }$. Therefore, their influence is already covered by a standard gust response factor approach [13], [14].

Nevertheless, also aerodynamic influences on the shape of the cycle count distribution have been observed. Mainly the shape coefficient $k$ of the long-termed wind statistic is to mention in this regard. In the investigations, both parameters of the Weibull modell for the long-termed wind statistic $A$ and $k$ have been modified. In Fig. 3 the regression parameter $\alpha$ and $\beta$ are plotted for a wide range range of $A$ - $k$-pairs. Additionally, 18 pairs of Weibull distributions, representing existing German locations and assuming different roughness lengths $z_{0}$ have been marked in this plot.

It becomes clear that the parameter of the Weibull distribution have a significant influence on the regression function of the $N_{\text {Life }}$ histograms. In order to evaluate the influence on the actual fatigue damage, the obtained cycle count distribution have been used with a linear damage accumulation strategy (modified Miner Rule) and based on following assumptions:

- As a quasi-static maximum stress $\Delta \sigma_{\max }=300 \mathrm{~N} / \mathrm{mm}^{2}$ is assumed

- The slopes for the S-N-curve are assumed with $m_{1}=3$ und $m_{2}=5$

- A cut-off level of dynamic stress of $\Delta \sigma_{L}=25,5 \mathrm{~N} / \mathrm{mm}^{2}$ is considered, below this level no damage growth is considered

In Fig. 4, the effect of different locational wind statistics on the actual fatigue damage is plotted for two different structural notch cases $\Delta \sigma_{C}$.

\subsubsection{Dynamic Properties of the considered Structure}

It is well known, that the dynamic structural properties affect the maximum response stress $\Delta \sigma_{\max }$ of a structure considering the gust response behavior. Using the presented stochastic approach, it is furthermore of interest, to which extent these properties do effect the shape of the long-termed cycle count distribution as well. For this aim, a parameter study has been 


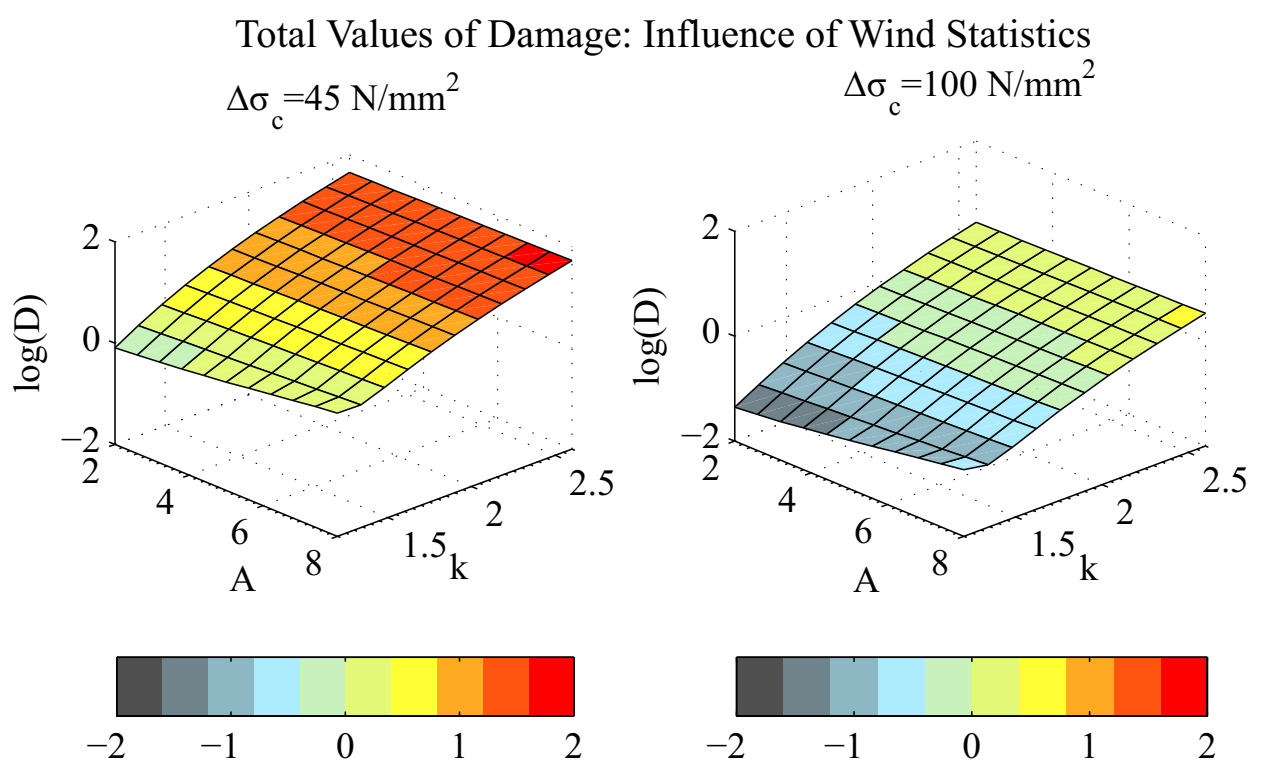

Figure 4: Influence of Long-Term Distribution of Wind Speeds on the Fatigue Damage

performed, in which both parameters eigenfrequency and damping ratio have been changed within structurally relevant limits. The influence on the regression parameter $\alpha$ and $\beta$ of the $N_{\text {Life }}$ function has been plotted in Fig. 5 for a specific pair of Weibull parameters. It becomes apparent that both dynamic properties are of significant importance considering the distribution of cycles.

Again, the influence on the actual fatigue damage has been considered under the same assumptions than in section 4.2.1. In Fig. 6, the associated absolute values of damage are plotted.

\subsection{Bilinear Regression of Cycle Count Distributions for various Systems}

In the preceding section mainly four parameters have been identified, which affect the shape of the cycle count distribution. These parameters are:

- Eigenfrequency $f$

- Logarithmic Damping $\delta$

- Scale Parameter $A$ of the Weibull distribution of wind speeds

- Shape Parameter $k$ of the Weibull distribution of wind speeds

In order to enable a combined consideration of all these parameters, besides exemplary calculations (like those presented in Fig. 5), a wide matrix of Weibull pairs $A$ - $k$ has been investigated in order to achieve a broader database concerning the influences on the cycle count distributions. All in all, over 14.000 dynamic calculations have been performed. For a tight provision of these results, in the following a reduction of the 4-dimensional problem is described. The two-dimensional result surfaces for $\alpha$ and $\beta$ (in the $f-\delta$-plane) of each Weibull-pair can be fitted by an appropriate shape function with a least-squares method (s. Fig. 2). Subsequently, the parameters of the chosen shape function can be plotted dependent on $A$ and $k$. Hence, the 4-dimensional problem has been broken down into two 2-dimensional problems, which can be easily plotted in surface diagrams.

The mathematical separation of the 4-dimensional result matrices for $\alpha$ and $\beta$ succeeds with introduction of the auxillary matrices $\kappa$ and $\lambda$ and the shape function $\Omega$ : 


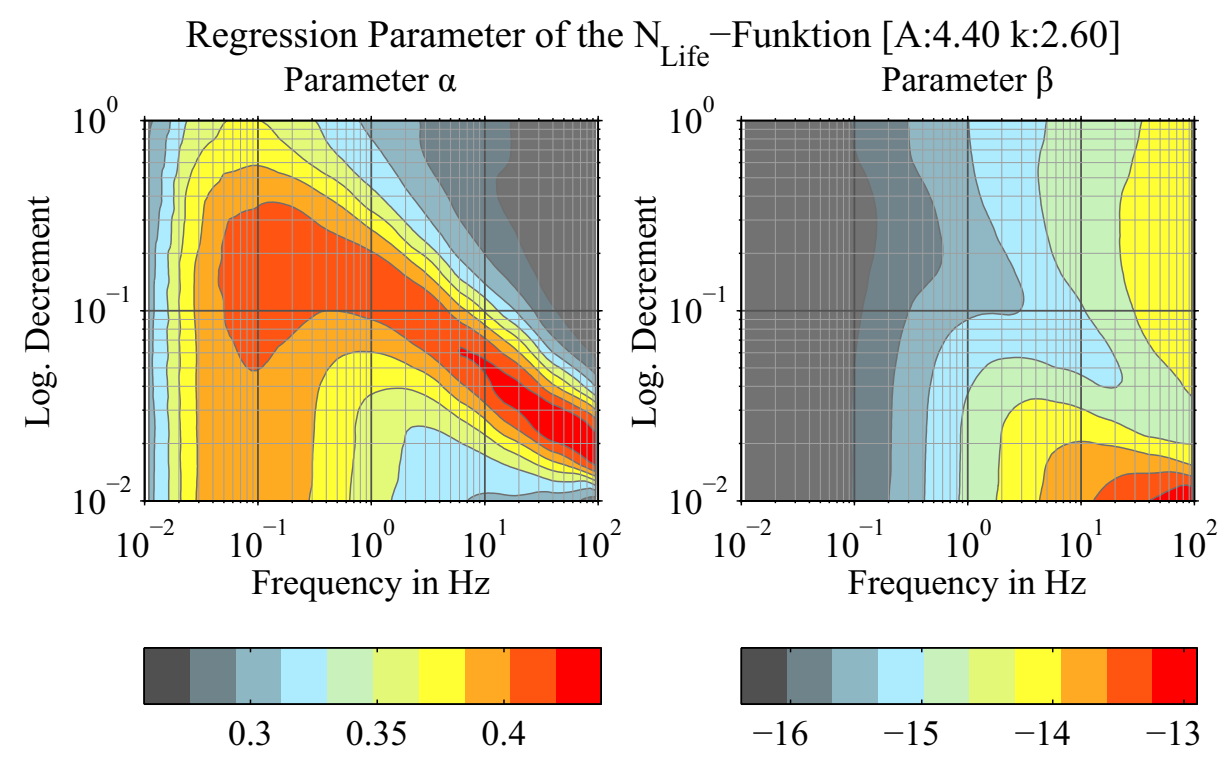

Figure 5: Influence of structural dynamic properties on the cumulative cycle count distribution $N_{L i f e}$ within 50 years

$$
\begin{aligned}
\alpha(f, \delta, A, k) & =\kappa(A, k) \cdot \Omega(f, \delta) \\
\beta(f, \delta, A, k) & =\lambda(A, k) \cdot \Omega(f, \delta)
\end{aligned}
$$

Different shape functions have been investigated. As a most detailed approach, a biquadratic polynominal with 6 parameters has been used. Further simplifications showed, that the number of necessary parameters could be reduced to 3, representing a simplified bilinear approach:

$$
\Omega(\delta, f)=c_{1} \cdot \log \delta+c_{2} \cdot \log f+c_{3}
$$

Using a two-dimensional optimization according to the least-squares method allowed the description of the surface with the mentioned parameters:

$$
\sum_{i} \sum_{j}[\Omega(\delta, f)-\Psi(f, \delta, A, k)]^{2} \rightarrow \min
$$

Where $\Psi$ represents the result matrices for $\alpha$ and $\beta$. Accordingly, the conditional equations are given by:

$$
\begin{aligned}
\alpha & =\kappa_{1}(A, k) \cdot \log \delta+\kappa_{2}(A, k) \cdot \log f+\kappa_{3}(A, k) \\
\beta & =\lambda_{1}(A, k) \cdot \log \delta+\lambda_{2}(A, k) \cdot \log f+\lambda_{3}(A, k)
\end{aligned}
$$

The planar representation of the regression results of the $N_{L i f e}$-distribution showed partially appreciable deviations between the exact calculation and the approximation to the bilinear shape functions, see Fig. ??. This was mainly caused by the fact that there is a dependency between the variables $\alpha$ and $\beta$, so the choice of a slightly larger $\alpha$-value leads to a slightly smaller $\beta$ value and vice versa. With regard to the optimization strategy, this means that there may be no clear optima, and the local minimum of Eq. (15) lies in a shallow valley and thus the optimization found is not particularly stable. Therefore, a second constrained optimization step has been 


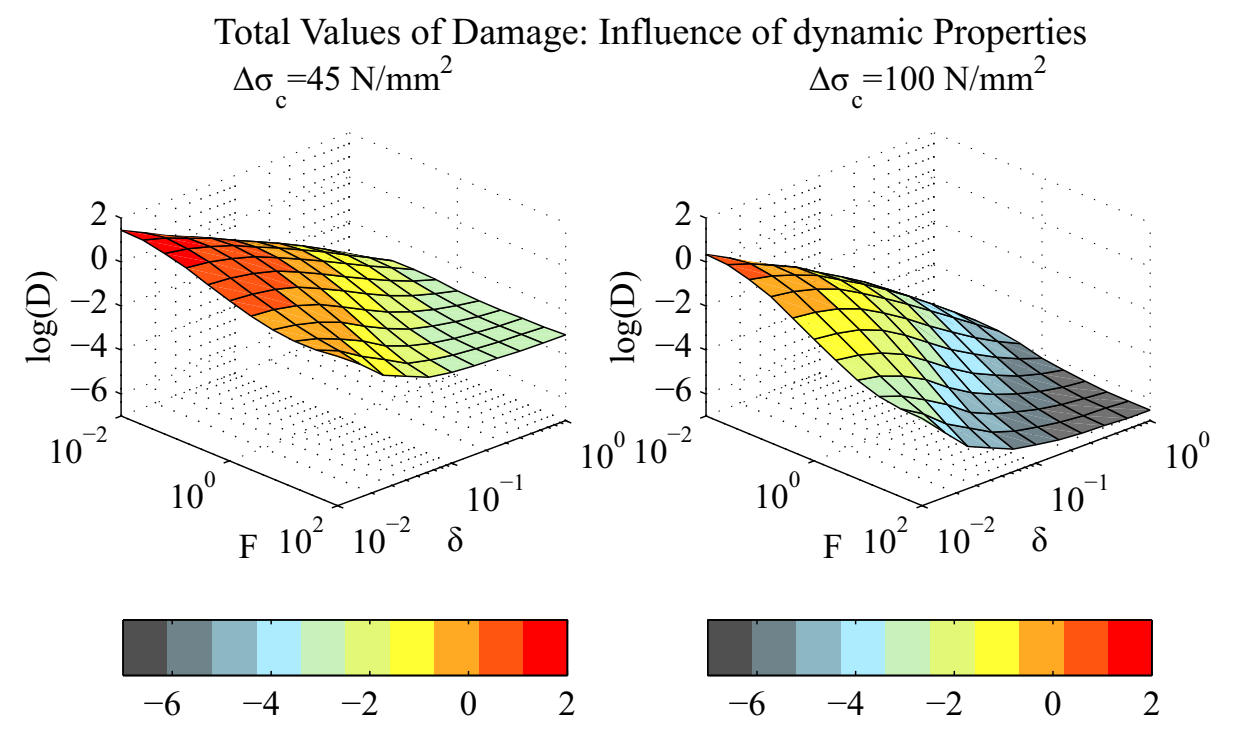

Figure 6: Damage values $\log (D)$ for an exemplary structure dependent on dynamic properties and considered notch case $\Delta \sigma_{C}$

made. In this step, the former linearized results for $\alpha$ have been kept and the corresponding, optimum values for $\beta$ have been determined.

The final results of the bi-linear optimization of all considered dynamic calculations are plotted in Fig. 8. In combination with Eq. (16) and (17) the values $\kappa_{i}$ and $\lambda_{i}$ can be used directly to determine a site and structure related cycle count distribution.

\section{DAMAGE EQUIVALENCE FACTOR CONCEPT}

In the preceding sections the main computation strategy and a simplified possibility for the determination of realistic cycle count distributions is presented. The latter allows a detailed verification of the actual fatigue life considering appropriate $\mathrm{S}-\mathrm{N}$ curves and damage accumulation strategies. However, at least for a pre-design stage, the damage assessment might be still too extensive, as the damage integral has still to be solved. For this reason some additional considerations have been made, allowing to reduce the fatigue assessment to a really simple check of the fatigue endurance. The following items are addressed in order to allow a further simplification:

- Consideration of a realistic shape of the $N_{L i f e}$-curve based on $\alpha(f, \delta, A, k)$ and $\beta(f, \delta, A, k)$

- Assumption of a certain shape of the structural S-N curve (trilinear or single slope)

- Linear damage hypothesis (elementary or modified Miner rule)

Based on these preconditions, an equivalent stress amplitude $\Delta \sigma_{E}$ at a given number of cycles can be computed which leads to an identical damage than the original collective. The ratio between the maximum stress amplitude $\Delta \sigma_{\max }$ and $\Delta \sigma_{E}$, is therefore denoted as a damage equivalence factor.

In order to calculate the damage due to the $N_{\text {Life }}$ cycle count distribution in a general form, the density of cycle numbers $N_{\text {Life }}^{\prime}(s)$ in accordance to Eq. (7) is needed. The deviation of the cumulative cycle count function is given by: 


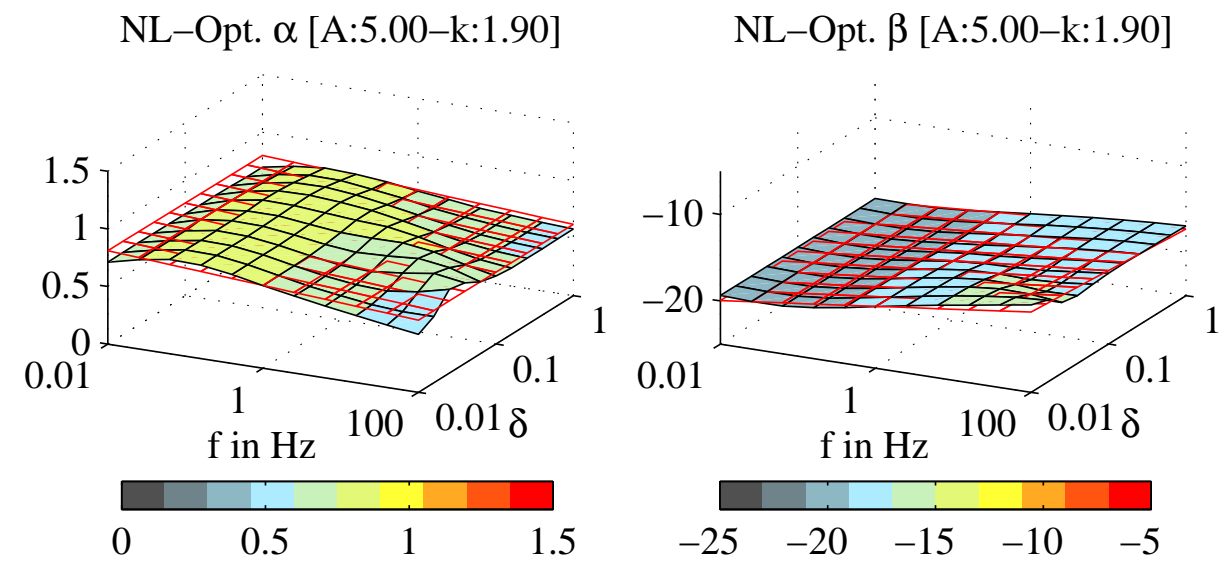

Figure 7: Exemplary fitting of the computation results with a bilinear shape function (Weibull parameter: $A=5,0$ $\mathrm{m} / \mathrm{s}$ and $\mathrm{k}=1,9$ ) after first optimization step

$$
N_{\text {Life }}^{\prime}(s)=\frac{d N_{\text {Life }}(s)}{d s}=-\frac{\ln (10)}{\gamma(s)} \cdot \exp \left[-\frac{\beta+\gamma(s)}{2 \alpha} \cdot \ln (10)\right]
$$

where:

$$
\begin{aligned}
\gamma(s) & =\sqrt{\beta^{2}-400 \cdot \alpha+4 \cdot s \cdot \alpha} \\
s & =\Delta \sigma / \Delta \sigma_{\max }
\end{aligned}
$$

The general formulation of the structural damage under consideration of the elementary Miner rule leads to:

$$
D=\int_{\Delta \sigma} \frac{N_{L i f e}^{\prime}(\Delta \sigma)}{N_{c} \cdot\left(\frac{\Delta \sigma_{c}}{\Delta \sigma}\right)^{m}} d \Delta \sigma
$$

where $\Delta \sigma_{c}$ is the notch case of the structural detail and $m$ ist the slope of the S-N curve. As the $N_{\text {Life }}$ curve represends the cumulative cycle counts within 50 years, Eq. (19) yields to the associated damage within this period.

\subsection{Elementary Miner-Rule (acc. to Palmgren)}

In this section the steps are described, which allow the development of damage equivalence factors using the elementary Miner rule. The equivalent stress range $\Delta \sigma_{E}$ can be calculated implicitly by the definition of the number equivalent of cycles $n_{E}=N_{D}=5 \cdot 10^{6}$ and by the demand of an identity of damages:

$$
\frac{n_{E}}{N_{E}}=\frac{N_{D}}{N_{E}} \stackrel{!}{=} D_{\text {Miner,elementary }}
$$

The associated stress range amplitude $\Delta \sigma_{E}$ is defined based on the structural S-N curve:

$$
\Delta \sigma_{E}=\left(\Delta \sigma_{D}^{m} \cdot \frac{N_{D}}{N_{E}}\right)^{\frac{1}{m}}
$$


Scale Parameter A in $\mathrm{m} / \mathrm{s}$
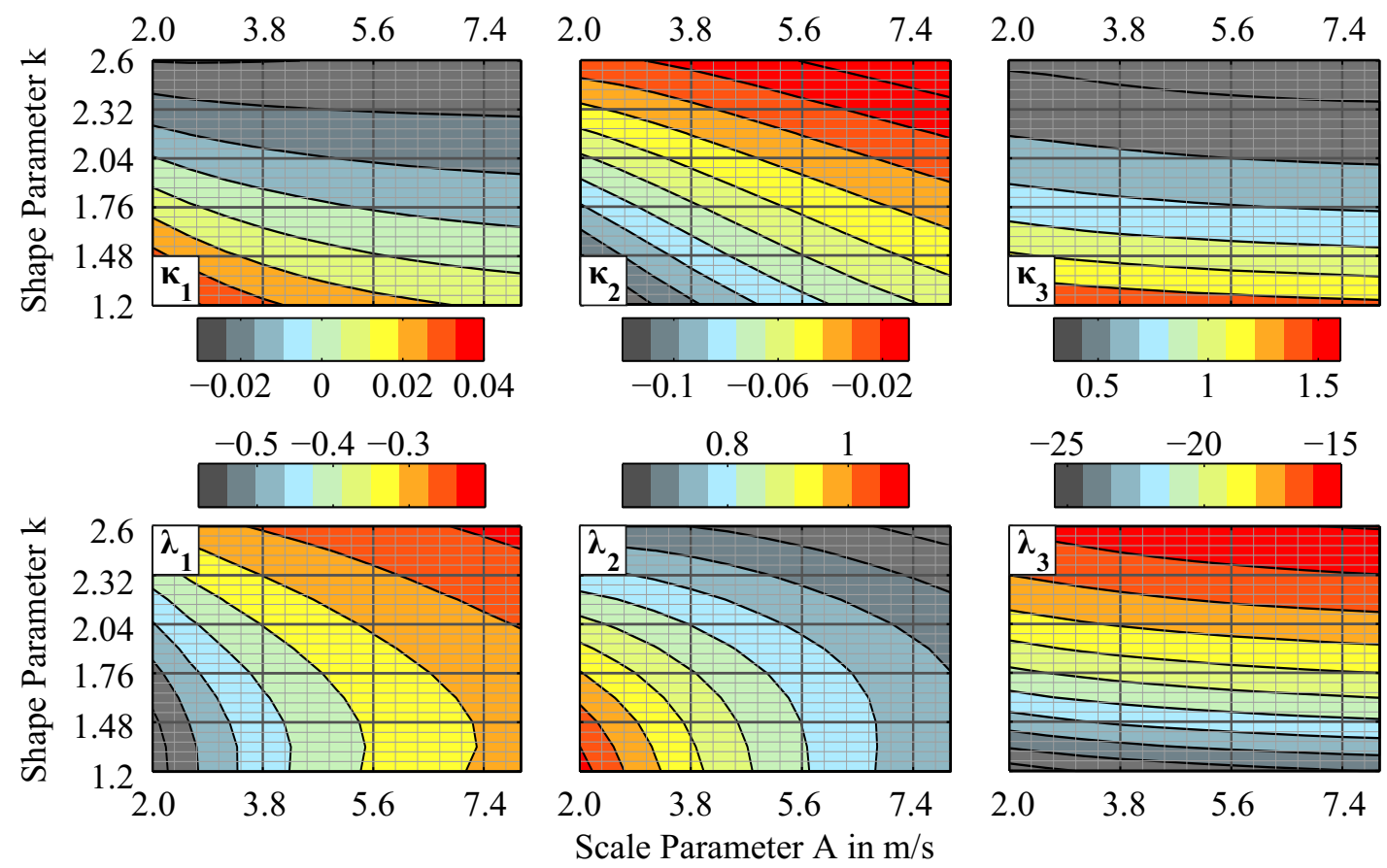

Figure 8: Parameter $\kappa$ and $\lambda$ for the simplified determination of realistic cycle count Spectra considering dynamic structural properties and the wind statistic on site

and hence, if the damage $D$ is known:

$$
\Delta \sigma_{E}=\left(\Delta \sigma_{D}^{m} \cdot D\right)^{\frac{1}{m}}
$$

Under consideration of Eq. (19), the equivalent stress amplitude can finally be derived to:

$$
\Delta \sigma_{E}=\left[\frac{1}{N_{D}} \cdot \int_{0}^{\infty} N_{\text {Life }}^{\prime}\left(100 \cdot \frac{s}{\Delta \sigma_{\max }}\right) \cdot s^{m} d s\right]^{\frac{1}{m}}
$$

The damage equivalence factor $K_{F}$ for the consideration of the shape of the stress range collective is given by:

$$
K_{F}=\Delta \sigma_{\max } / \Delta \sigma_{E}
$$

The fatigue endurance verification can be performed considering characteristic values as follows:

$$
\Delta \sigma_{\max } / K_{F} \stackrel{!}{\leq} \Delta \sigma_{D}
$$

A realistic damage assessment is enabled using the bearable cycle counts $N_{E}$, which are associated with the stress amplitude $\Delta \sigma_{E}$ :

$$
N_{E}=\left(\frac{\Delta \sigma_{D}}{\Delta \sigma_{E}}\right)^{m} \cdot N_{D}
$$



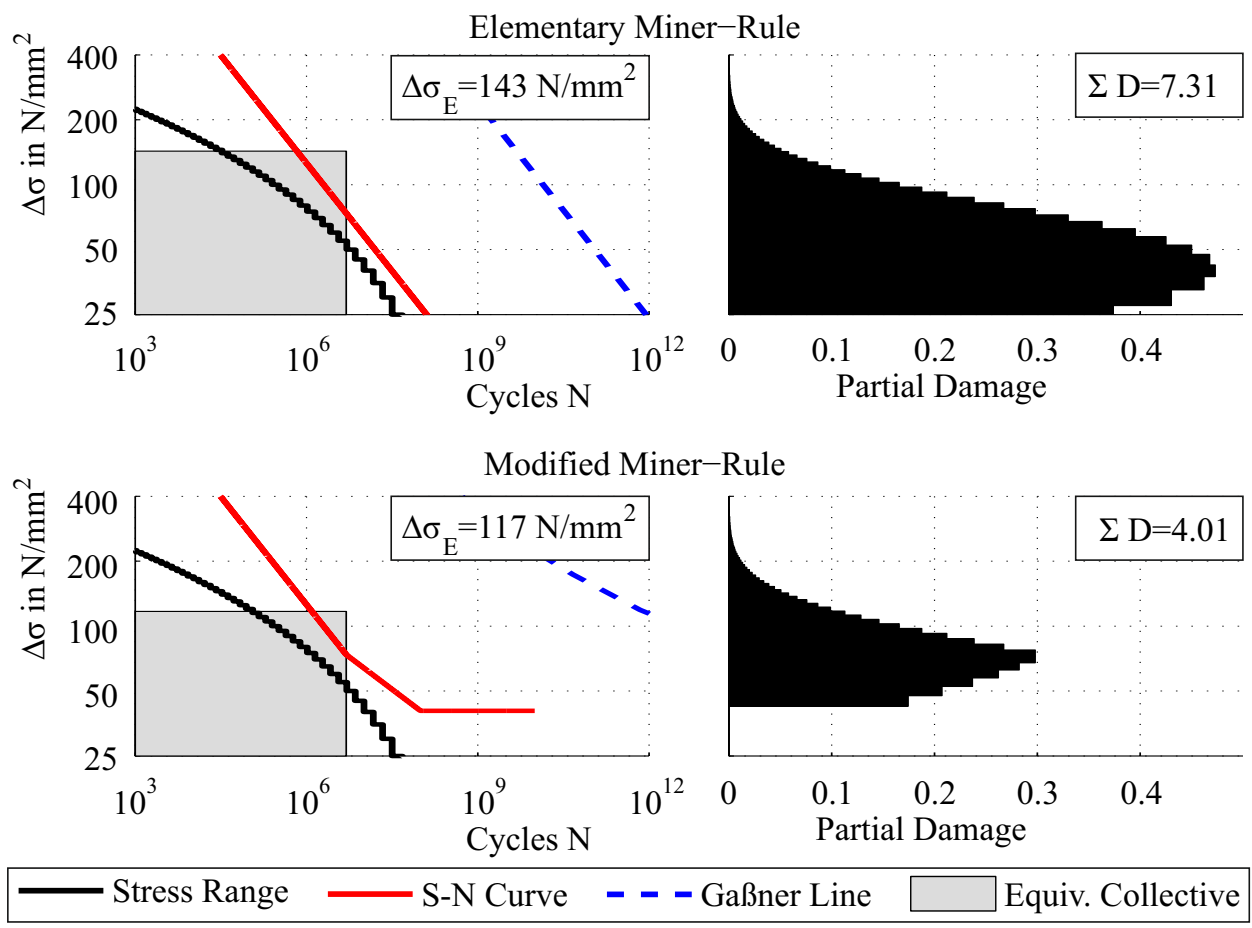

Figure 9: Computation of partial and cumulative Damage for a $N_{L i f e}$-function with $\alpha=0.7$ and $\beta=-17.4$ and $\Delta \sigma_{\max }=500 \mathrm{~N} / \mathrm{mm}^{2}$ considering a notch case $\Delta \sigma_{C}=100 \mathrm{~N} / \mathrm{mm}^{2}$ according to elementary and modified Miner rule

Finally, the corresponding damage value is defined as:

$$
D=\frac{n_{E}}{N_{E}}=\frac{N_{D}}{N_{E}}
$$

The verification now includes implicitly the individual shape of the stress and leads to the same collective damage value as the integral of the actual collective consideration based on Eq. (19).

\subsection{Modified Miner-Rule (acc. to Haibach)}

The derivation of the damage equivalence factor $K_{F}$ is slightly more complex, when a S$\mathrm{N}$ curve $\Delta \sigma_{W}(N)$ with different slopes is considered. Depending on the particular slope, the equivalent stress amplitude $\Delta \sigma_{E}$ can be determined by a distinction of cases to:

$$
\begin{array}{ll}
\Delta \sigma_{E}=\left(\Delta \sigma_{D}^{m_{1}} \cdot D\right)^{\frac{1}{m_{1}}} & \text { for } N_{E}<N_{D} \\
\Delta \sigma_{E}=\left(\Delta \sigma_{D}^{m_{2}} \cdot D\right)^{\frac{1}{m_{2}}} & \text { for } N_{D} \leq N_{E}
\end{array}
$$

Using the modified Miner rule in the damage calculation, the value of $D$ is is generally lower, but for most materials more realistic than according to Eq. (27). For the further computation, Eq. (24) till Eq. (27) remain valid.

\subsection{Damage Equivalence Factors for Application}

For an enabling of a preferably simple and general fatigue assessment concept, it was essential to avoid dependencies of the damage equivalence factor $K_{F}$ to other parameters than the shape of the cycle count distribution. Especially an influence of the maximum stress amplitude 


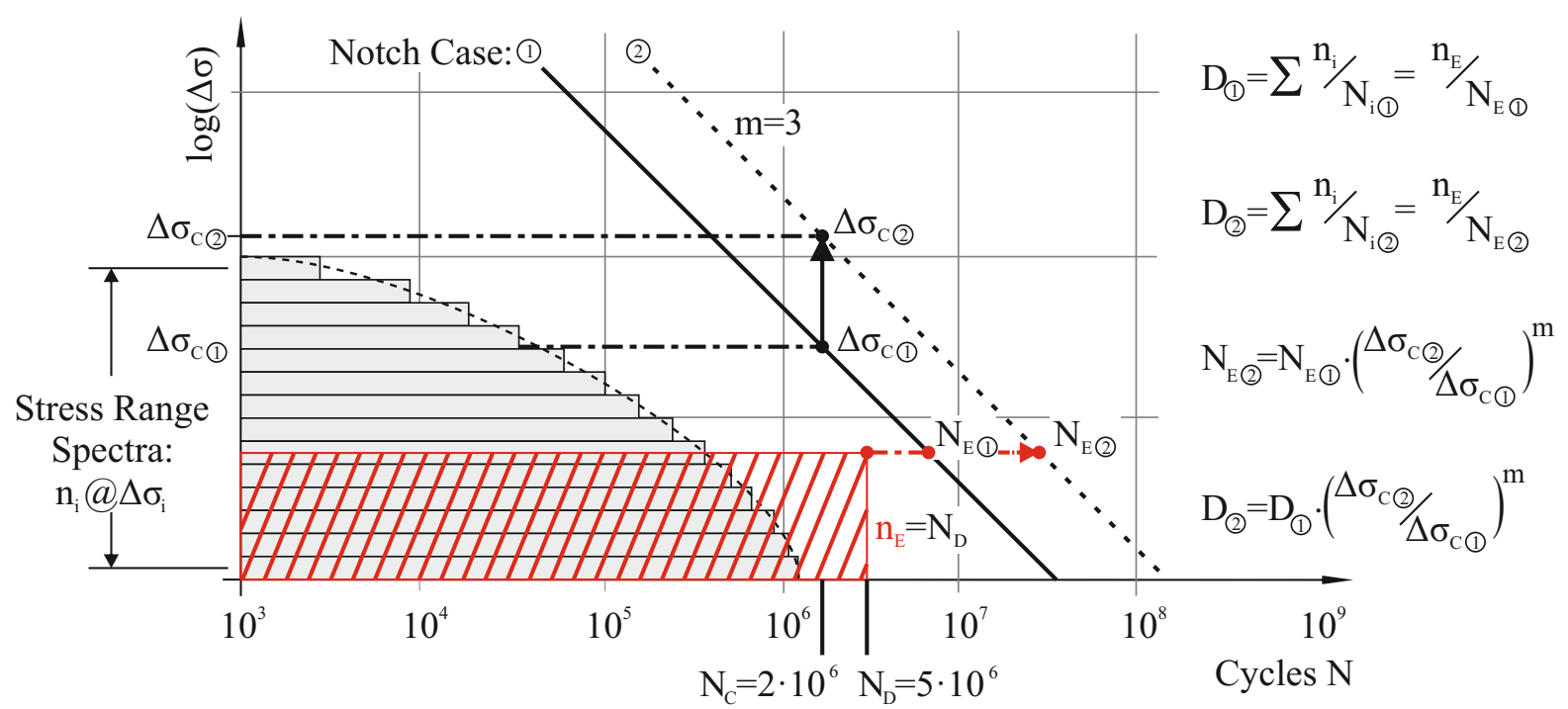

Figure 10: Reduction of a stress range histogram to a damage equivalent block histogram and independence of $\Delta \sigma_{E}$ on the notch case $\Delta \sigma_{C}$ under consideration of the elementary Miner rule

$\Delta \sigma_{\max }$ and the notch case $\Delta \sigma_{C}$ would complicate the concept. Considering Eq. (22), it becomes apparent that the equivalent stress range depends on the fatigue endurance limit $\Delta \sigma_{D}$ in a direct way and indirectly by the damage $D$. Therefore, the equivalent stress range $\Delta \sigma_{E}$, and thus the damage equivalence factor $K_{F}$, are not dependent on the notch case $\Delta \sigma_{C}$. However, as soon as a S-N curve with different inclinations is used, this independency is lost, due to the necessary case distinction according to Eq. (28) and (29).

Generally, the maximum value of the stress collective $\Delta \sigma_{\max }$ affects the computed structural damage. In case of a simple linear S-N curve (elementary Miner rule) it follows for different collective maximums $\Delta \sigma_{\max , 1}$ and $\Delta \sigma_{\max , 2}$ :

$$
D_{2}=D_{1} \cdot\left(\frac{\Delta \sigma_{\max , 2}}{\Delta \sigma_{\max , 1}}\right)^{m}
$$

Consequently, in conjunction with equation (22) it follows, that the equivalent stress range $\Delta \sigma_{E}$ acts linear to the maximum stress amplitude $\Delta \sigma_{\max }$ of the collective. Thus, the damage equivalence factor $K_{F}$ is also independent of the collective maximum amplitude $\Delta \sigma_{\max }$.

When the modified Miner's rule is used, the damage equivalence factor $K_{F}$ depends on both the collective maximum and the notch case of the S-N curve. For the establishment of an easy concept it is therefore advantageous to use the conservative elementary Miner rule with a unique slope of $m_{1}=3$.

In Fig. 11, the damage equivalence factors are plotted for arbitrary combinations of the regression parameter $\alpha$ and $\beta$ of the cycle count distribution $N_{\text {Life }}$. Considering the influence of wind characteristic and structural dynamic properties, the equivalent stress factors can be read directly from the chart. Therefore, the bi-linear regression model from Section 4.3 has been used. As the plot is restricted to three different values for the shape parameter $k$ of the Weibull distribution, the usage is limited to rather approximative calculations, for example as a part of a pre-dimensioning. For the accurate determination of the damage equivalent factors it is recommended to determine the parameter $\alpha$ and $\beta$ in advance using the equations described in section 4.3. 


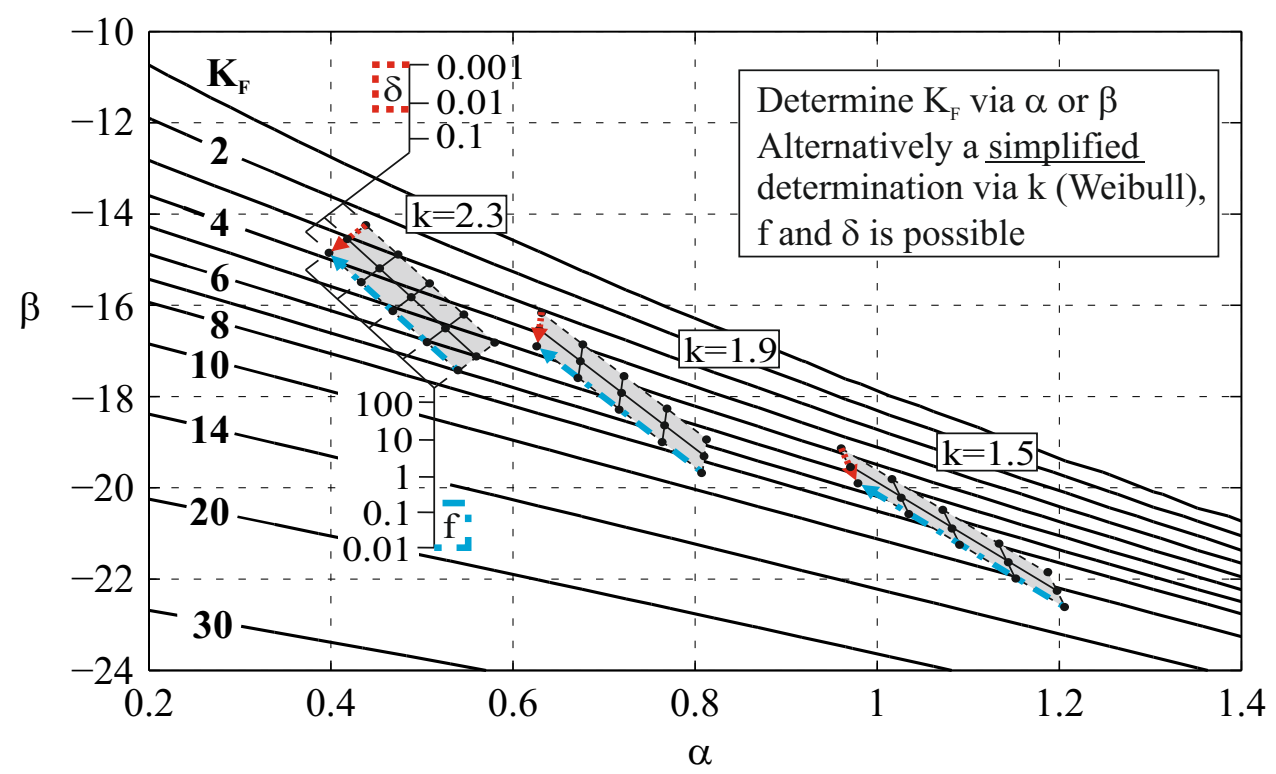

Figure 11: Damage Equivalence Factors $K_{F}$ dependent on the regression parameter of the $N_{L i f e}$-function and assuming the elementary Miner rule

For the fatigue assessment finally Eq. (25) can be simplified to:

$$
\gamma_{F f} \cdot \frac{\Delta \sigma_{\max }}{K_{F}} \stackrel{!}{\leq} \frac{\Delta \sigma_{D}}{\gamma_{M f}}
$$

Due to the implicit form of the elementary Miner rule this proof is generally on the safe side. The corresponding damage value can be calculated as follows:

$$
D=\left(\frac{\Delta \sigma_{D}}{\Delta \sigma_{\max }} \cdot K_{F}\right)^{-m}
$$

\section{EXAMPLE}

In this section the presented methods are demonstrated briefly using the example of a steel chimney, which is located at the Institute for Steel Structures, see Fig. 12. Several years ago, Gahlemann conducted measurements on-site and he calculated a fatigue Damage, which is associated with measured the gust response behavior of the structure [5]. In his investigations he computed a maximum dynamic stress amplitude of $\Delta \sigma_{\max }=142.9 \mathrm{~N} / \mathrm{mm}^{2}$ and a damage value of $D_{\text {Gust }}=0.21$ within a period of 50 years using the modified Miner rule.

Considering the structural and site dependent aerodynamic parameter, given in Fig. 12, a damage equivalence factor of approximately $K_{F}=6$ can be extracted from Fig. 11. For the notch case of $\Delta \sigma_{C}=36 \mathrm{~N} / \mathrm{mm}^{2}$, the fatigue endurance limit derives to $\Delta \sigma_{D}=26.5 \mathrm{~N} / \mathrm{mm}^{2}$. Based on these values a damage of:

$$
D=\left(\frac{26.5}{142.9} \cdot 6.0\right)^{-3}=0.72
$$

is estimated. Taking into account, that the simplified approach is based on the elementary Miner concept, the correlation of the damage values is quite impressive. 


\section{CONCLUSIONS}

It has been shown, that reliable fatigue verifications based on cumulative damage predictions are possible by means of closed form spectral methods. Due to the tight manageability of the presented formulas, the procedure is advisable for the fatigue verification of arbitrary structures under gusty wind loads.

As main influencing parameters of the stress cycle count distribution, mainly the structural frequency, the damping behavior and the wind statistic on site has been identified. Under consideration of appropriate shape function, the four independent parameters have been brought together in order to enable a simplified, graphical description.

Finally, the damage equivalence factor concepts has been introduced on the base of the regression parameters which describe the actual shape of the cycle count distribution. In combination with the elementary Miner rule it was possible to calculated divisors, which allow a reduction of the maximum stress level in order to consider the distribution of cycle counts. The methodology is very simple in use and it is demonstrated for an exemplary structure.

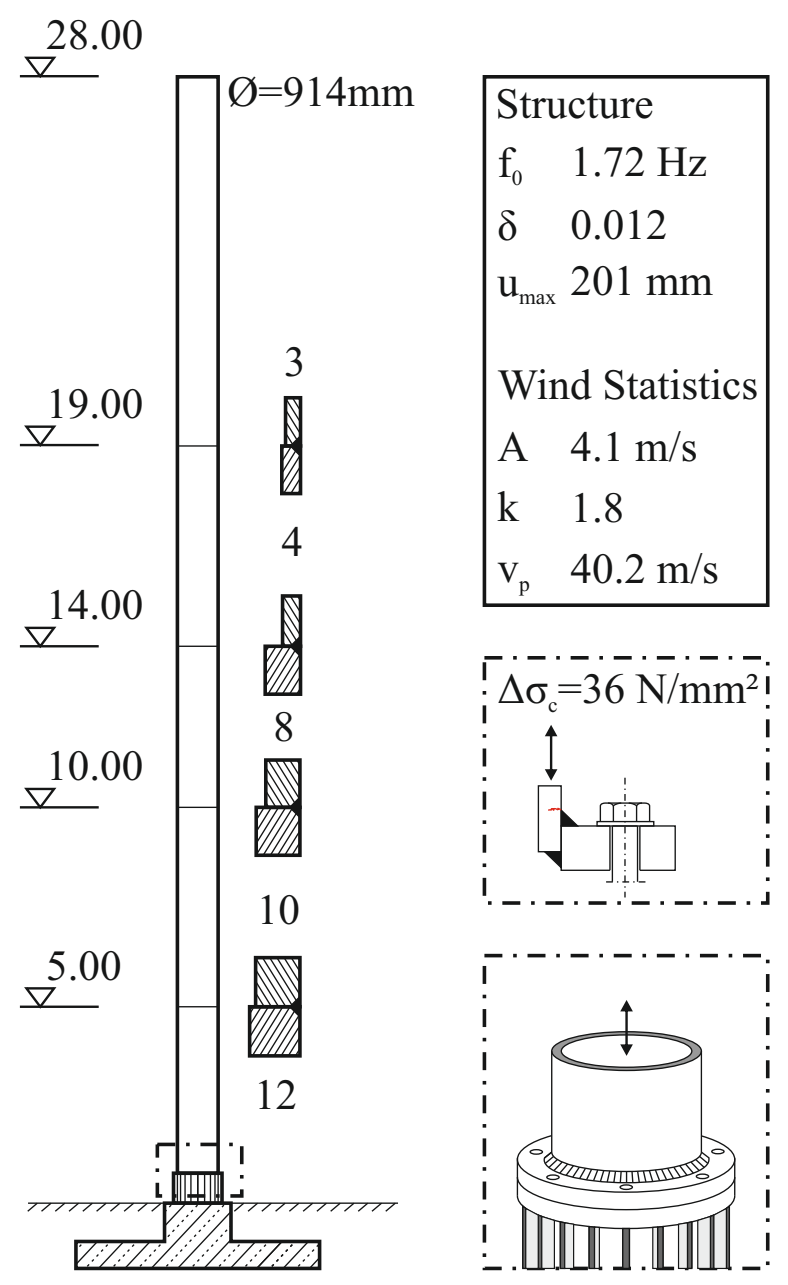

Figure 12: Drawing of the Steel-Chimney and considered Notch case 


\section{REFERENCES}

[1] J. S. Bendat. Probability functions for random responses: Prediction of peaks, fatigue damage and catastrophic failures. NASA Technical Report, (CR-33), 1964.

[2] Alan G. Davenport. The buffeting of structures by gusts. In Teddington Middlesex National Physical Laboratory, editor, Wind effects on Buildings and Structures, volume 1, pages 357-391. Her Majesty's stationery office, 1965.

[3] T. Dirlik. Application of computers to fatigue analysis. PhD thesis, Warwick University, Warwick, 1985.

[4] Europäisches Komitee für Normung. Eurocode 3: Bemessung und konstruktion von stahlbauten teil 1-9: Ermüdung, 05.2005.

[5] Thomas Gahlemann. Messungen der dynamischen Windeinwirkungen auf einen 28m hohen Stahlkamin und Vergleich mit vorliegenden Berechnungsverfahren. PhD thesis, RWTH Aachen, Aachen, 1994.

[6] Andrew Halfpenny. Rainflow cycle counting and acoustic fatigue analysis techniques for random loading. In RASD2010. 2010.

[7] J. D. Holmes. Wind loading of structures. Spon Press, London and New York, 2001.

[8] F. H. Kemper. Böeninduzierte Schwingungsanfälligkeit von durchlässigen Fassadenelementen unter Berücksichtigung nichtlinearer Struktureigenschaften im Grenzzustand der Ermüdung. $\mathrm{PhD}$ thesis, Schriftenreihe des Instituts für Stahlbau, Heft 76, Shaker-Verlag, Aachen, 2013.

[9] F. H. Kemper and M. Feldmann. Appraisement of fatigue phenomena due to gust induced vibrations based on closed-form approaches. In ICWE 13. AMSTERDAM, 2011.

[10] F. H. Kemper and M. Feldmann. Fatigue life prognosis for structural elements under stochastic wind loading based on spectral methods: Part 1: Linear structures. In G. de Roeck, editor, Proceedings of the 8th International Conference on Structural Dynamics, EURODYN 2011, pages 1629-1635. Leuven, 2011.

[11] F. H. Kemper and M. Feldmann. Fatigue life prognosis for structural elements under stochastic wind loading based on spectral methods: Part 2: Nonlinear structures. In G. de Roeck, editor, Proceedings of the 8th International Conference on Structural Dynamics, EURODYN 2011, pages 1636-1643. Leuven, 2011.

[12] M. Matsuishi and T. Endo. Fatigue of metals subjected to varying stress. Japan Society of Mechanical Engineers, 1968.

[13] Giovanni Solari. Gust buffeting. i: Peak wind velocity and equivalent pressure. Journal of Structural Engineering, 119(2):365, 1993.

[14] Giovanni Solari. Gust buffeting. ii: Dynamic alongwind response. Journal of Structural Engineering, 119(2):383, 1993.

[15] Friedhelm Stangenberg, Rolf Breitenbücher, Otto T. Bruhns, Dietrich Hartmann, Rüdiger Höffer, Detlef Kuhl, and Günther Meschke. Lifetime-Oriented Structural Design Concepts. SpringerVerlag Berlin Heidelberg, Berlin and Heidelberg, 2009.

[16] I. Troen and E. L. Petersen. European wind atlas. Risø National Laboratory, Roskilde, 1989. 
[17] P. Watson and B.J Dabell. Cycle counting and fatigue damage. In Symposium on statistical aspects of fatigue testing. Warwick University, 1975.

[18] P. H. Wirsching and A. M. Shehata. Fatigue under wide band random stresses using rainflow method. Journal of Engineering Materials and Technology, pages 205-211, 1977. 